DOE/ER/40553-5

\title{
NUCLEAR TRANSPARENCY AND DOUBLE BETA DECAY OF MOLYBDENUM 100
}

\author{
Annual Progress Report \\ for Period February 1, 1994 - January 31, 1995 \\ H. W. Nicholson \\ Mount Holyoke College \\ South Hadley, Massachusetts 01075
}

July 1994

Prepared for

The U.S. Department of Energy under Grant No. DE-FG02-90ER40553

NOTICE

This report was prepared as an account of work sponsored by the United States Government. Neither the United States nor the United States Department of Energy, nor any of their employees, nor any of their contractors, subcontractors, or their employees, make any warranty, express, or implied, or assumes any legal liability or responsibility for the accuracy, completeness, or usefulness of any information, apparatus, product or process disclosed or represents that its use would not infringe privately owned rights. 


\section{DISCLAIMER}

This report was prepared as an account of work sponsored by an agency of the United States Government. Neither the United States Government nor any agency thereof, nor any of their employees, make any warranty, express or implied, or assumes any legal liability or responsibility for the accuracy, completeness, or usefulness of any information, apparatus, product, or process disclosed, or represents that its use would not infringe privately owned rights. Reference herein to any specific commercial product, process, or service by trade name, trademark, manufacturer, or otherwise does not necessarily constitute or imply its endorsement, recommendation, or favoring by the United States Government or any agency thereof. The views and opinions of authors expressed herein do not necessarily state or reflect those of the United States Government or any agency thereof. 


\section{DISCLAIMER}

Portions of this document may be illegible in electronic image products. Images are produced from the best available original document. 


\begin{abstract}
During the past year, work has been nearly completed on a Physical Review paper with the final results of a search for neutrinoless double- $\beta$ decay of molybdenum 100 with collaborators from the Lawrence Berkeley Laboratory, the University of New Mexico, and the Idaho Engineering Laboratory. A major part of this work has been to carry out a more extensive statistical analysis of the data.

During the spring of 1994 while he was on a one semester sabbatical leave from Mount Holyoke, Prof. Sean Sutton spent the majority of his time at Orsay, France working on NEMO 3, a next generation double- $\beta$ decay experiment involving molybdenum 100 and other isotopes.

Professors Nicholson and Sutton have designed and built a scintillating fiber hodoscope which has been used in the May-July AGS high energy physics run at Brookhaven Laboratory in AGS experiment 850 to measure color transparency. Professor Nicholson has had primary responsibility for the design, construction, and installation of this hodoscope and for overseeing the construction and installation of two scintillating counter upstream hodoscopes. To date, the fiber hodoscope has handled total beam rates exceeding $20 \mathrm{MHz}$ with beam rates as high as $10 \mathrm{MHz}$ on a single fiber. In addition, Prof. Nicholson has been regularly involved in taking shifts during the run.

Work to study light intensification and readout electronics to be used in the $\mathrm{CsI}(\mathrm{Tl})$ calorimeter in the SLAC B factory has just begun this summer. This work will be carried out in collaboration with University of Massachusetts faculty Professor Richard Kofler and his colleagues and students as part of a general B factory collaborative effort.

Dr. Ted Kycia of Brookhaven laboratory has also invited Professors Nicholson and Sutton to become involved in a neutrino oscillation experiment which he is proposing. This would involve constructing several triggered spark chamber detectors read out and digitized optically with CCD cameras one of which would be located most likely in western Connecticut or Massachusetts.
\end{abstract}




\section{NARRATIVE}

During the past year, Professors Nicholson and Sutton have continued to work on completing a final Physical Review paper on a molybdenum double$\beta$ decay experiment carried out in the Consil silver mine in Osburn, Idaho with collaborating physicists from Lawrence Berkeley Laboratory (LBL), the University of New Mexico, and the Idaho National Engineering Laboratory (INEL). The major part of this effort has been to carry out a more extensive statistical analysis on the data which was published in Physical Review Letters in August, 1993. ${ }^{1}$ This analysis has been complicated by a lack of well defined and universally agreed upon statistical techniques for carrying it out, but every effort is being made to complete this paper and submit it to the Physical Review in the fall of 1994.

Along with the work underway to complete the LBL, University of New Mexico, INEL, Mount Holyoke double- $\beta$ decay experiment, Professor Sutton has continued to collaborate with Professor Nicholson on an experiment to study color transparency at the Brookhaven Laboratory AGS described below. He has also become an active collaborator in the NEMO 3 international collaboration. (NEMO stands for Neutrinoless Experiments with Molybdenum.) This collaborative experiment, which has been funded by the French government, is designed to be a new generation experiment to study rare decays of molybdenum 100 as well as other double- $\beta$ decay isotopes.

The NEMO 3 detector, which will be operated in the Fréjus Underground Laboratory near Modane, France, is briefly described in Chapter 9 of the NEMO proposal given in the Appendix of this report. It has the general shape of a large, vertical cylinder. The source material is a foil or part of a foil 3 meters high and 2 meters in diameter. Specially designed Geiger tubes which use sense wires as the outside electrode and which are oriented vertically on either side of the foil provide tracking information for decays originating in or outside the foil. A scintillator calorimeter gives energy measurements on the tracks with a FWHM resolution of about $16 \%$ at $1 \mathrm{MeV}$ and time of flight resolution of 7 nanoseconds. A solenoid magnet producing a .003 Tesla longitudinal field helps to identify pair production events and externally incident electrons. A detailed description of the detector is given in the complete NEMO proposal, a document in which Professor Sutton had

\footnotetext{
${ }^{1}$ M. Alston-Garnjost et al., Phys. Rev. Lett. 71, 831 (1993)
} 
a major role in editing during the past year. He also gave a 45 minute talk on the NEMO 3 detector at the July 1994 Snowmass Conference.

Professor Sutton has received support for travel from the National Science Foundation for this project and spent the majority of a half-year sabbatical leave at Orsay working on the NEMO 3 project and contributing to the data analysis of the earlier NEMO 2 experiment. (Some results of the NEMO 2 experiment are given in the Appendix of this report.) At present, he and physicists from ITEP in Moscow have the responsibility for developing techniques for purifying the $10 \mathrm{~kg}$ of molybdenum 100 source material available from Russia to radioactive impurity levels between 10 and 100 parts per trillion and then characterizing the type and amounts of any residual radioactive impurity elements.

In June, 1994, at a collaboration meeting to discuss purification which Professor Sutton organized at Orsay, procedures for preliminary studies were worked out. Dr. John Baker from INEL, who chemically purified the 150 gms of molybdenum 100 used in the LBL, Mt. Holyoke, New Mexico, INEL double- $\beta$ decay experiment described above to residual radioactive material levels well below 1 part per billion and who attended this meeting (along with Professor Nicholson), is now working with Professor Sutton to study the problems of scaling up his process to purify $10 \mathrm{~kg}$ of molybdenum 100 and characterize the final product. In addition, Al Smith, who operates the low level counting facility at LBL and who has a large ( $110 \%$ efficient) germanium detector 600 feet underground at Oroville dam with the sensitivity to detect residual amounts of radioactivity approaching the required levels, has agreed to count some of this molybdenum before and after the purification process.

Professor Nicholson's principal efforts during the past year have been his continuing involvement in AGS experiment 850 being carried out at Brookhaven Laboratory to study color transparency in nuclei for incident beam particle momenta between 6 and $20 \mathrm{GeV} / \mathrm{c}$. This experiment is briefly described in a Workshop of Future Directions in Particle \& Nuclear Physics at Multi-GeV Hadron Facilities (Brookhaven Laboratory, March 1993) paper included in the Appendix of this report.

Professor Nicholson has been the person with primary responsibility for designing, obtaining components, and building a scintillator fiber beam hodoscope which is placed just upstream of the experimental targets while Professor Sutton was primarily responsible for determining how to build a support structure to integrate the fiber detector with the inner straw tube 
detector and target holder. The actual construction of the detector was carried out primarily at Mount Holyoke. This hodoscope, which is $2.54 \mathrm{~cm}$ wide and $5.08 \mathrm{~cm}$ high and has 23 overlapping vertical and 48 overlapping horizontal channels, is used to tune the beam, is part of.the experimental trigger, and, when used in conjunction with an upstream beam hodoscope designed and constructed at Brookhaven Laboratory by Steve Gushue, can be used to determine incident beam particle trajectories. A more detailed description of this hodoscope and some preliminary testing carried out last year is described in a Scifi Conference (Notre Dame, October 1993) paper included in the Appendix to this report. Actual beam efficiency measurements for the 48 horizontal fibers (which measure the vertical extent of the beam) obtained during the AGS May-July 1994 run are given in Figure 1 and the efficiency for the 23 vertical fibers are shown in Figure 2. Efficiencies are less than $100 \%$ because of gaps between fibers and the method used to measure on line efficiencies, but when this is taken into account the overall efficiency for the working fiber channels is about $90 \%$. A later run when most the fiber channels were working close to maximum efficiency and which shows the beam profile is given in Figure 3 .

The EVA detector has worked reasonably well during this running period and to date data has been collected at $6.0,7.5,9.0$ and $12.0 \mathrm{GeV} / \mathrm{c}$. pp elastic scattering can clearly be seen in the $6.0 \mathrm{GeV} / \mathrm{c}$ online data analysis (Figure 4). Work is currently underway to obtain better straw tube calibration data to improve the momentum and position resolution of tracks in the detector.

Professor Nicholson has also become involved with the calorimeter design for the SLAC B factory detector. He has been involved with the editing of Chapter 8 in the Letter of Intent (SLAC-443) and is currently investigating the performance of waveshifter plates and Hamamatsu PIN photodiodes (3588-01) which are being considered for reading out the calorimeter CsI(Tl) crystals. This work, which will be carried out in collaboration with Prof. Richard Kofler and his colleagues and students at the University of Massachusetts Amherst, will be part of a long term effort to contribute to the construction of the final 10,000 crystal calorimeter which will most likely be distributed among many of the $\mathrm{B}$ factory's collaborating institutions.

Finally, Professors Nicholson and Sutton have been invited to collaborate on an experiment to study neutrino oscillations which is being proposed by Dr. Ted Kycia of Brookhaven Laboratory. This experiment, if approved, would involve constructing several detectors, each consisting of steel plates 
and optical spark chambers read out with CCD cameras, one of which would be located in western Connecticut not far from the Five College area. Although this experiment is only at the proposal state at this time, it is one which would be well suited by geography and interest to a Mount Holyoke collaboration.

Although both Professor Nicholson and Professor Sutton are nominally involved in all of the activities described above, at this time Professor Sutton has taken the principal role in the NEMO 3 collaboration and has made some significant contributions to the EVA experiment, while Professor Nicholson has been principally involved in the EVA experiment and in the early stages of the $\mathrm{B}$ factory collaboration. Because of the relatively small DOE supported research program at Mount Holyoke there will almost certainly continue to be an overlap of involvements in all ongoing projects with each faculty member having one major current collaborative involvement. 


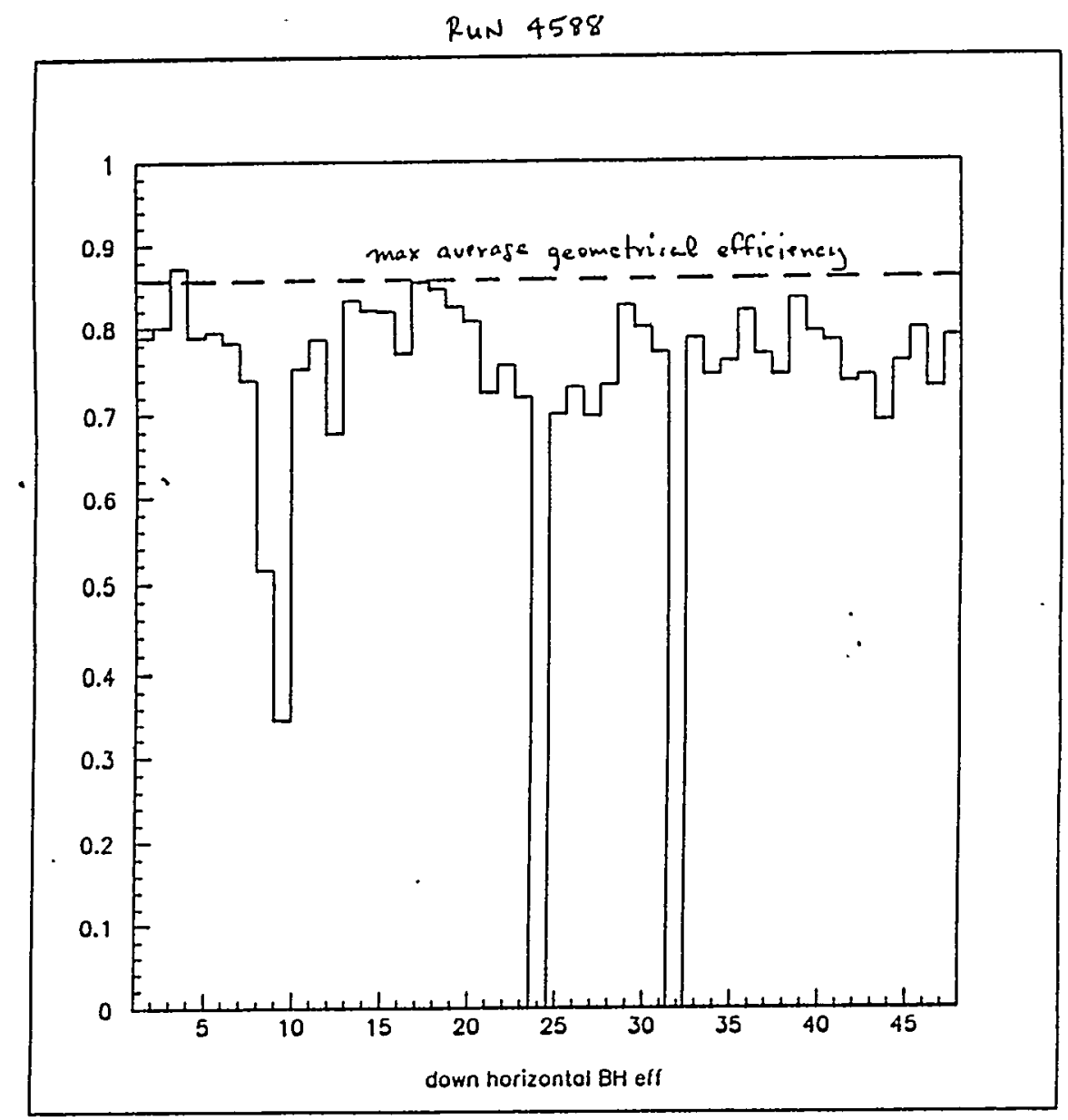

Figure 1

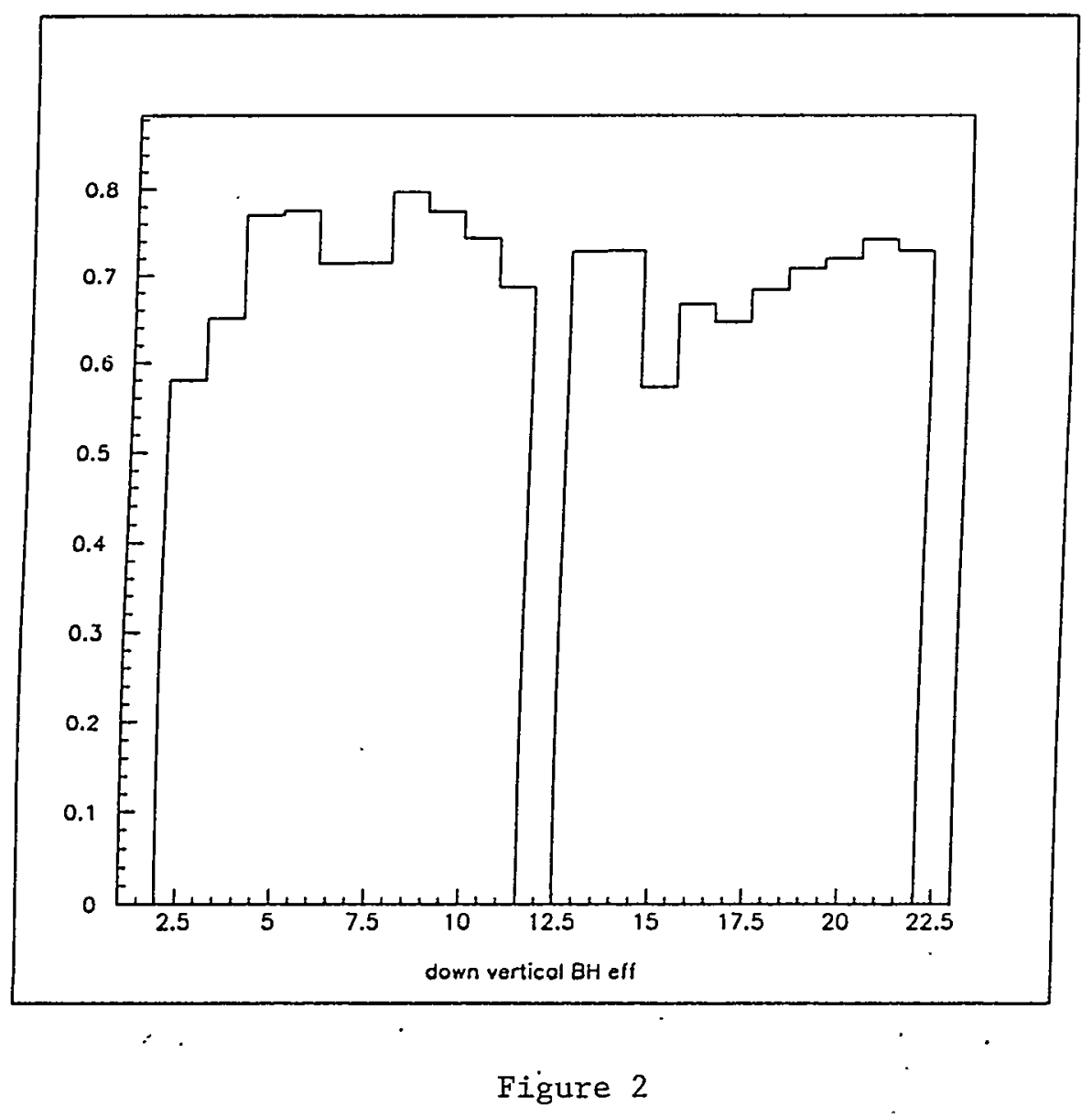




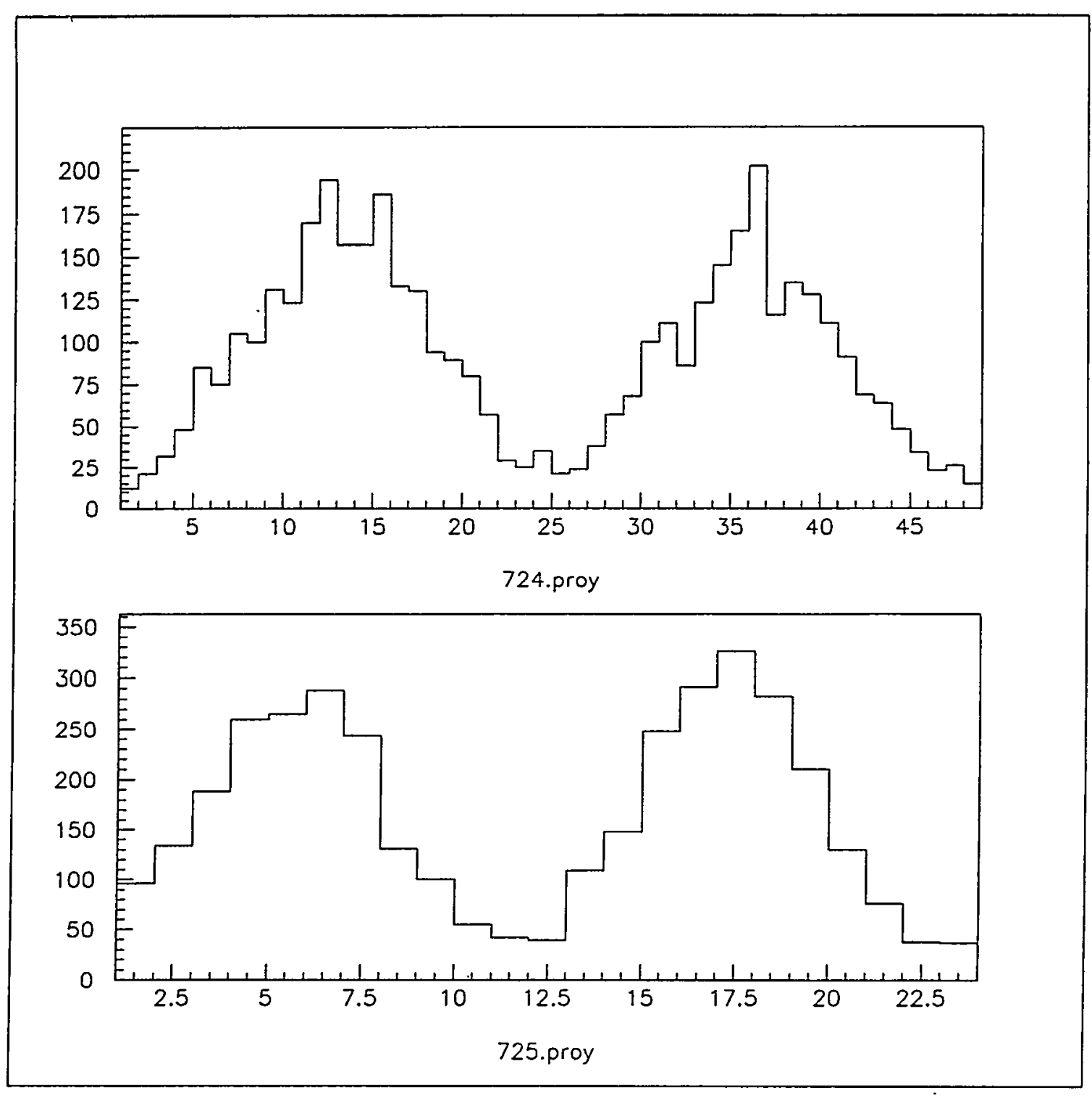

Figure 3

Vertical and horizontal beam profiles in downstream hodoscope. 
24

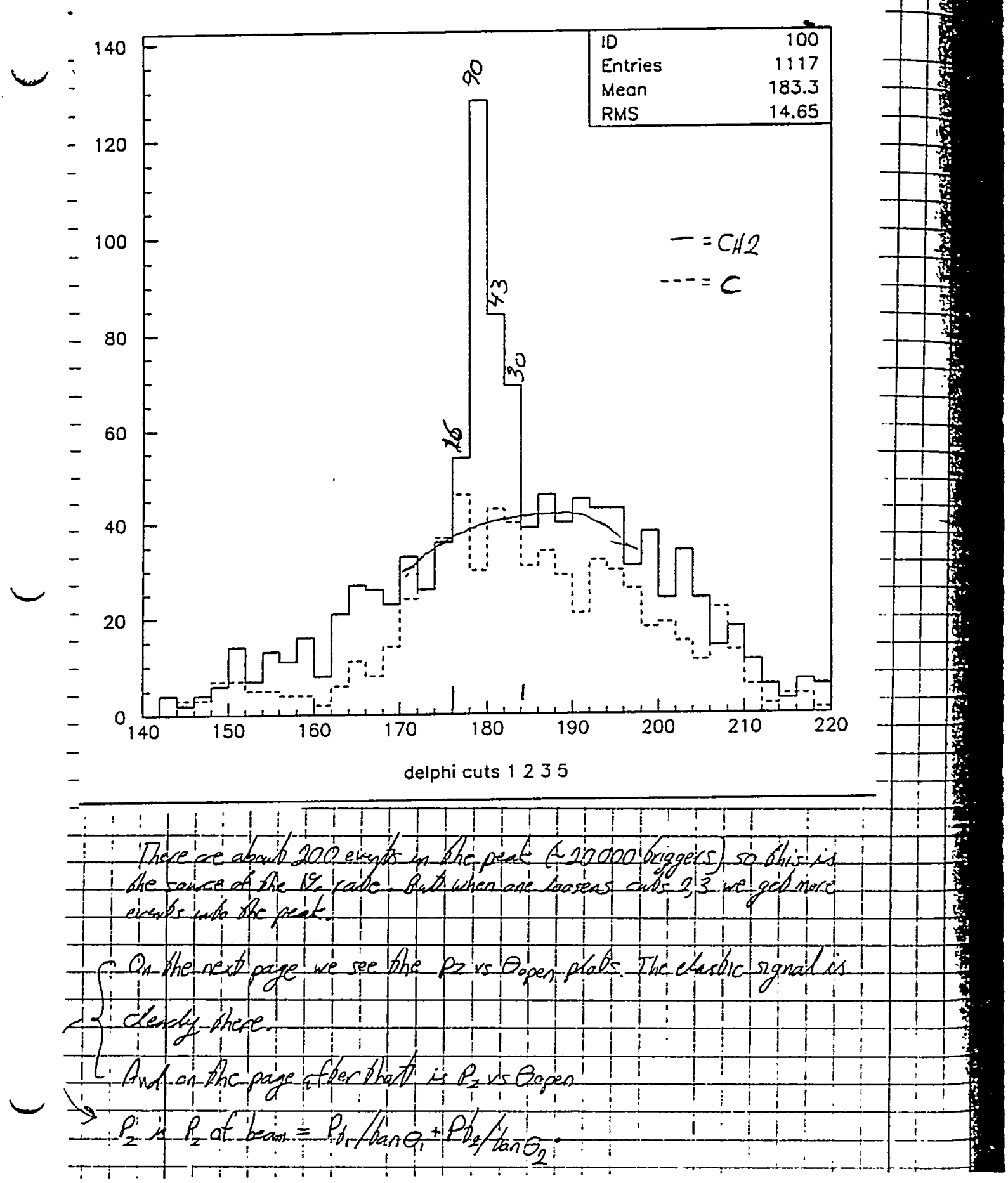

Figure 4

Online analysis showing pp elastic scattering peak at $6.0 \mathrm{GeV} / \mathrm{c}$ 


\section{APPENDIX}




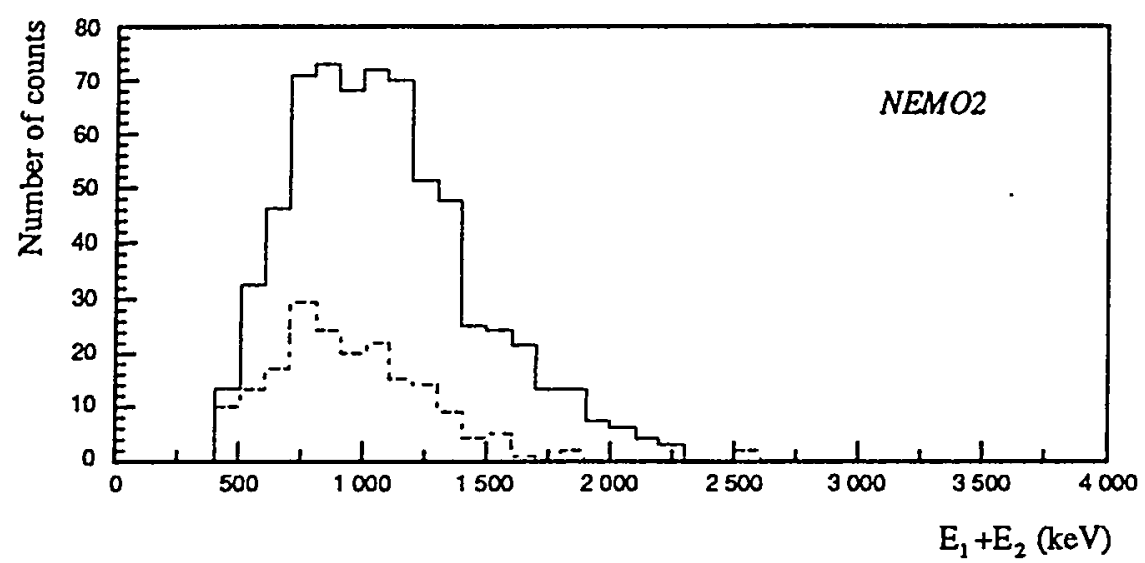

Figure 6.18: $2 e$ energy spectra in enriched and natural molybdenum (Phase III). The shapes of the spectra are different, one spectrum is dominated by the $\beta \beta 2 \nu$ process (solid line) and the other by external background (dashed line).

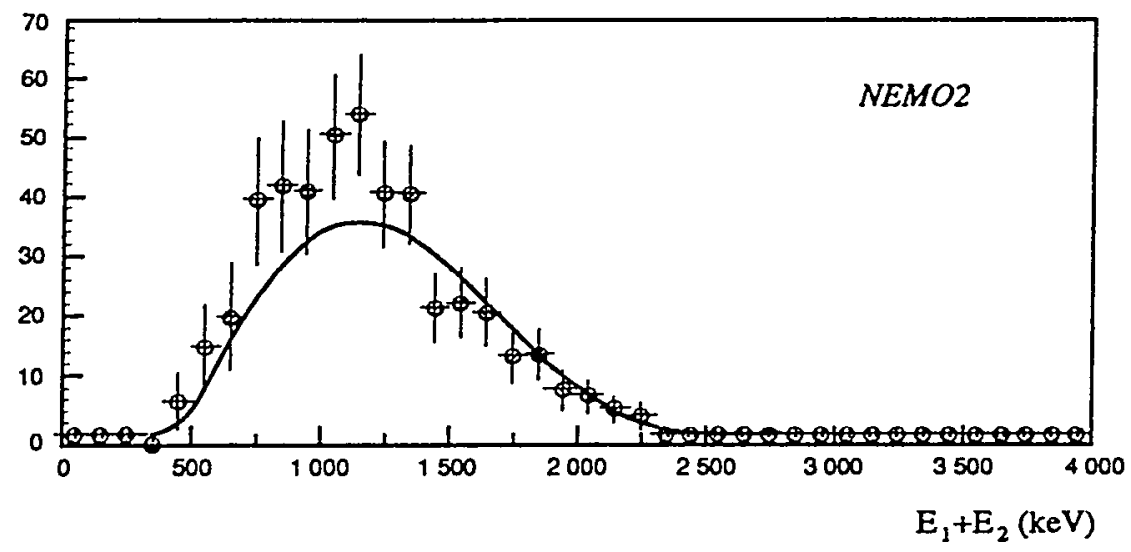

Figure 6.19: Fit of $\beta \beta 2 \nu$ signal of ${ }^{100} \mathrm{Mo}$ (Phase III). Error bars take into account the external background. Note that the uncertainty is larger in the low energy part of the spectrum. 


\section{Chapter 7}

\section{Conclusion}

In the current generation of experiments in double-beta decay, data most be taken continuously over the course of several years so that the detectors reliability and stability are well understood. NEMO 1 and NEMO 2 have run for 20,000 hours with nearly troublefrcc opcration. The tcchnical design, which was selected to track low energy electrons in a transparent gas detector, has provided fine three-dimensional information and can be incorporated into a larger detector. The advantage of having all the wires of the drift cell in the same direction allows for the efficient use of a cylindrical geometry in the proposed experiment. The time and energy measurements with plastic scintillators and photomultipliers are reliable and the calibration process is well understood.

The analysis of the NEMO 1 data shows that the background coming from decays outside of the detector is readily identified, in particular, the $\gamma$ ray flux with energies up to $8 \mathrm{MeV}$ can be explained by thermal neutron captures in the copper frame of the detector. Accordingly, shielding studies have been carried out to optimize the materials used. In the NEMO experiments a combination of lead and iron provide an effective attenuation of $\gamma$-rays and the neutron fluxes.

The analysis of the NEMO 2 data was done on a more complete set of channels (1e, $2 e, e \gamma, e \gamma \gamma, e \gamma \alpha)$ for which the results are the following.

- No two-electron events were observed with energies above $2.2 \mathrm{MeV}$ coming from a very pure (OFHC) cooper foil.

- A clear signal from the $\beta \beta 2 \nu$ process has been recorded using a pure $172 \mathrm{~g}$ enriched molybdenum metallic foil. No events were observed above $2.5 \mathrm{MeV}$ in this sample.

- The NEMO 2 detector is able to measure and accordingly select very pure metallic source foils with levels of contamination in ${ }^{214} \mathrm{Bi}$ and ${ }^{208} \mathrm{Tl}$ at the limit of $0.5 \mathrm{dpm} / \mathrm{kg}$ and $0.1 \mathrm{dpm} / \mathrm{kg}$, respectively. Finally, the search for one-electron events allows us to put some limits on the contamination of radioactive isotopes which only emit $\beta$ s. Therefore, the device is able to measure the levels of radioactivity in itself.

- The presence of external neutrons has been studied by exposing the outside of the experiment's shield to a neutron source. The energy spectrum of the two track events observed beyond $3 \mathrm{MeV}$ are due to $e^{+} e^{-}$pairs produced in the source foil. These events can be rejected by charge recognition in a magnetic field.

- The presence of radon in the ambient air of the laboratory strongly suggests that future detectors should be isolated from the radon by some purging process. 


\section{Chapter 9}

\section{Simplified description}

\subsection{Source}

The general layout of the NEMO 3 detector is shown in Fig. 9.1. The source is a cylinder $2.8 \mathrm{~m}$ in diameter, $2 \mathrm{~m}$ in height and $55 \mu \mathrm{m}$ thick. It is segmented into 30 sectors which permits flexibility in servicing the detector and access to patchwork source foils. Currently the proposed source foil is $10 \mathrm{~kg}$ of ${ }^{100} \mathrm{Mo}$. Note that monitoring the external background can again be done with an OFHC copper "source" foil as in NEMO 2 (Sec. 6.2).

\subsection{Tracking}

The Geiger drift cells (Sec. 5.1.1) are the same as in NEMO 2 but the length of the cells are increased by a factor of three. All the cells are vertical and as such parallel to the axis of the cylinder.

The previous prototypes demonstrated that the longitudinal coordinate can be measured by the plasma propagation time induced in the Geiger regime. The accuracy in the transverse and longitudinal position measurements are the same as that measured in NEMO 1 and NEMO 2 (Sec. 5.1.4), $\sigma_{T}=0.5 \mathrm{~mm}$ and $\sigma_{L}=5 \mathrm{~mm}$.

The transparency of the tracking device filled with helium gas and $2 \%$ ethyl alcohol will be improved by changing the field wire diameter from $100 \mu \mathrm{m}$ (nickel) to $30 \mu \mathrm{m}$ (iron).

\subsection{Energy and time-of-flight measurements}

Encrgy and time-of-flight measurements will be performed as in NEMO 2 (Sec. 5.3 and Sec. 5.4) by plastic scintillators covering the two vertical surfaces of the active tracking volume. To further enhance the acceptance efficiency, the end caps will be equipped with scintillators in the spaces between the Geiger cell layers.

The photon detection efficiency will be improved by increasing the thickness of the plastic scintillator ( $10 \mathrm{~cm}$ instead of $2 \mathrm{~cm}$ as in NEMO 2). The front face of the scintillator $(20 \mathrm{~cm} \times 20 \mathrm{~cm})$ is larger than in NEMO $2(12 \mathrm{~cm} \times 12 \mathrm{~cm})$ corresponding to a compromise between resolution and an affordable number of PMTs. The expected energy and time resolution (FWHM) is $16 \%$ and 700 ps at $1 \mathrm{MeV}$, respectively. 


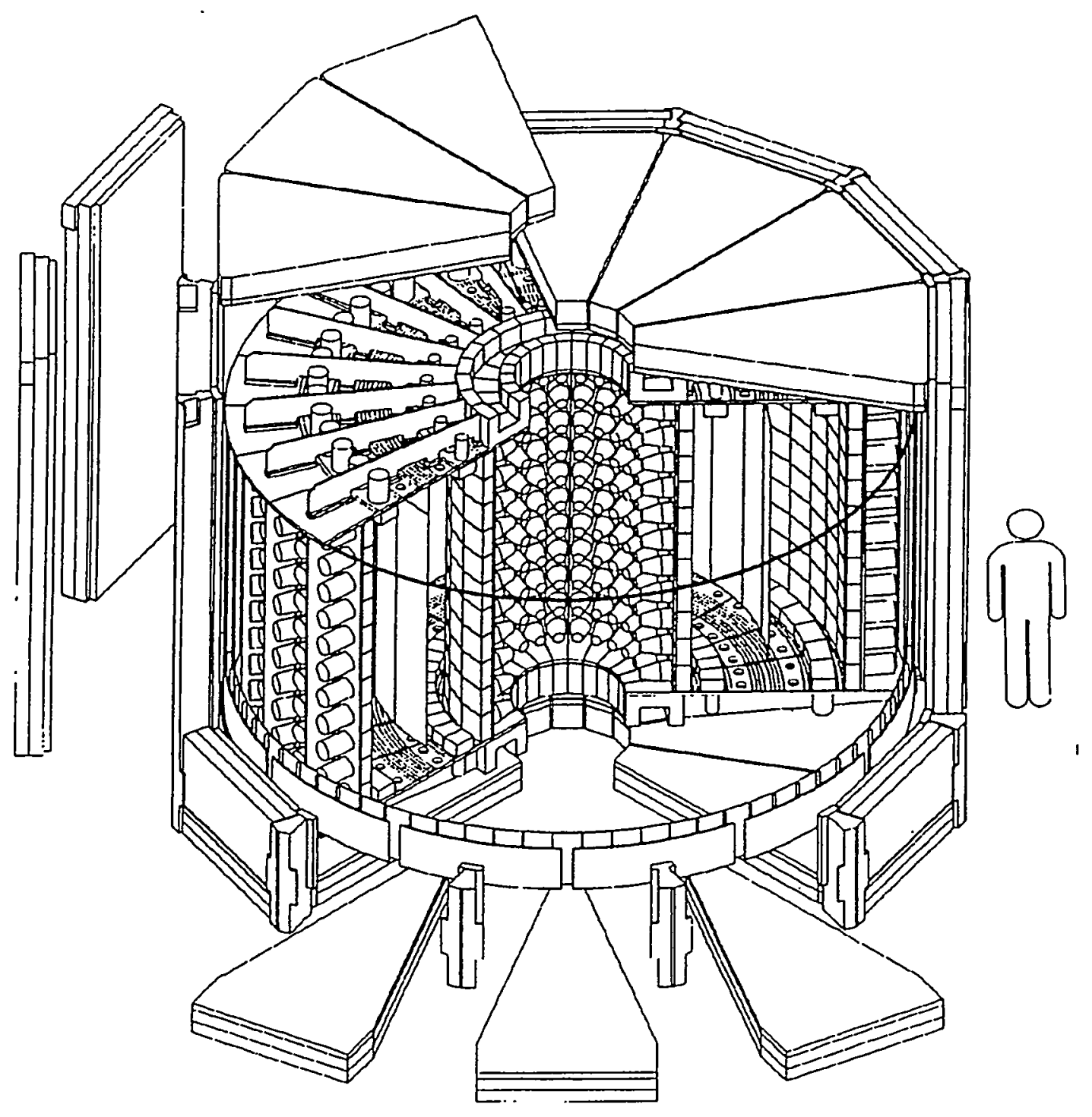

Figure 9.1: General layout of NEMO 3 


\subsection{Magnetic field}

As it has been shown in NEMO 1 and NEMO 2 with neutron sources (Sec. 6.2.2 and Sec. 6.7), the high energy $\gamma$-rays induced by neutron capture in the detector create $e^{+} e^{-}$ pairs in the 3 to $8 \mathrm{MeV}$ energy region. The 30 Gauss magnetic field induces a unique signature for the $e^{+} e^{-}$events. Additionally, the magnetic field will provide an extra rejection factor for incoming electrons.

\subsection{Shielding}

Studies with NEMO 1 (Sec. 6.2.1) have shown an effective shield for the attenuation of $\gamma$-rays and neutrons is a combination of $5 \mathrm{~cm}$ of lead surrounded by $20 \mathrm{~cm}$ of low activity iron.

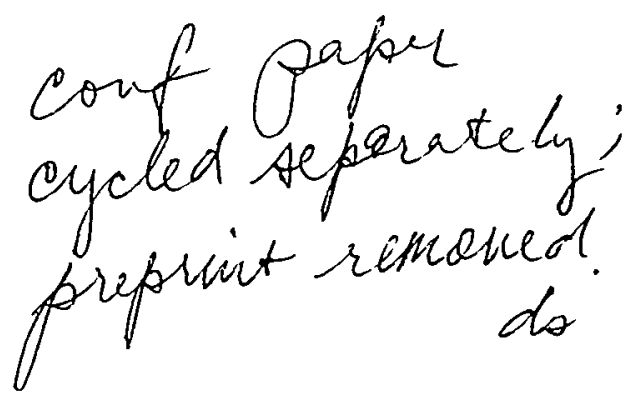

